\title{
Analytical modelling of the interpixel thermal crosstalk in superconducting edge-transition bolometer arrays
}

\author{
A Bozbey ${ }^{1}$, M Fardmanesh ${ }^{1,2}$, J Schubert $^{3}$ and M Banzet $^{3}$ \\ ${ }^{1}$ Electrical and Electronics Engineering Department, Bilkent University, Ankara 06800, \\ Turkey \\ ${ }^{2}$ Electrical Engineering Department, Sharif University of Technology, Tehran, Iran \\ ${ }^{3}$ ISG1-IT and Center of Nanoelectronic Systems for Information Technology, \\ Forschungszentrum Juelich GmbH, D-52425 Juelich, Germany \\ E-mail: bozbey@ieee.org and fardman@ee.bilkent.edu.tr
}

Received 15 February 2006, in final form 30 March 2006

Published 26 April 2006

Online at stacks.iop.org/SUST/19/606

\begin{abstract}
We present an analytical thermal model to explain the crosstalk in YBCO edge-transition bolometer arrays. The verification of the model was tested on sample array devices made of 200 and $400 \mathrm{~nm}$ YBCO films on $\mathrm{LaAlO}_{3}$ and $\mathrm{SrTiO}_{3}$ substrates. The model presented was able to explain the effects of the various physical parameters of the devices, such as the film thickness, operating temperature, and the device separation, which cause different response behaviours based on the variation of the related thermal crosstalk characteristics. In addition, the model is valid above the crosstalk-free modulation frequencies, where the effects of the thermal crosstalk on the response of the devices are negligible.
\end{abstract}

\section{Introduction}

There have already been a number of studies regarding the modelling of the response of single pixel bolometer devices [1-3] and bolometer arrays [4, 5]. However, a comprehensive model to explain the crosstalk phenomenon in the superconductive edge-transition bolometer arrays has not been reported. While modelling bolometer devices, two assumptions can be made. One of them, the large area bolometer assumption, assumes that the heat only propagates in the vertical direction, normal to the bolometer plane. The other assumption, used for modelling the crosstalk in the bolometer arrays, assumes that the heat mainly propagates in the lateral direction. Here, we combine the heat diffusion process in the vertical direction with the lateral heat diffusion process in the array plane to get the thermal diffusion model for the whole substrate. We explain the observed crosstalk based response of our devices, and compare the fitting results of the model with the experimental results of various samples of different separation distances, substrate materials, film thickness, and bias temperatures.

\section{Samples and experimental setup}

For verification of the analytical model, we prepared $4 \times 1$ bolometer arrays in $200 \mathrm{~nm}$ thick YBCO films deposited by pulsed laser deposition on crystalline substrates. We measured the thermal coupling or the crosstalk between the devices in the form of arrays of long bridges. The illuminated device in the array had an area of $20 \mu \mathrm{m} \times 1 \mathrm{~mm}$, and the neighbouring test devices had areas of $20 \mu \mathrm{m} \times 0.75 \mathrm{~mm}$. In order to measure the crosstalk between the devices, it is essential to keep the test bolometers optically isolated from the environment. It was also taken into consideration that optically isolating the devices does not cause additional thermal coupling artifacts in the array. The array configuration is shown in figures 1 (a) and (b). One bolometer, the 'source device', (named B) is illuminated with modulated IR radiation, whereas the remaining three bolometers, 'sense devices', are blocked with a free standing reflecting mask. The separation of the sense bolometers named, A, C, and D, from the source bolometer was 30, 50, and $150 \mu \mathrm{m}$, respectively. In the previously reported lateral diffusivity based simple model, we used the centre-to-centre distances between the bolometers [6]. However, in this model, edge-to-edge distances resulted in better fits. 
(a)

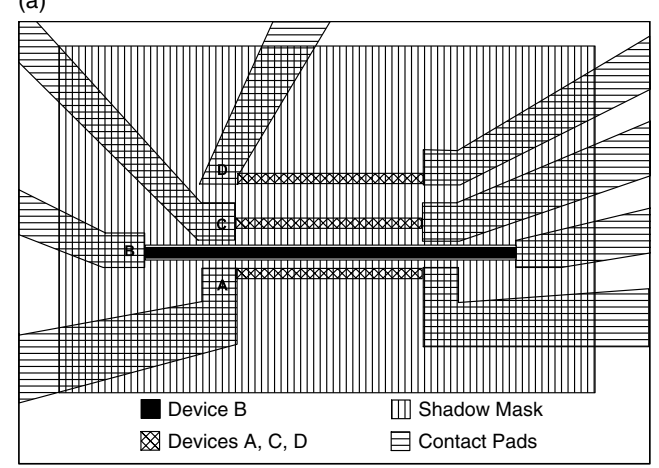

(b)

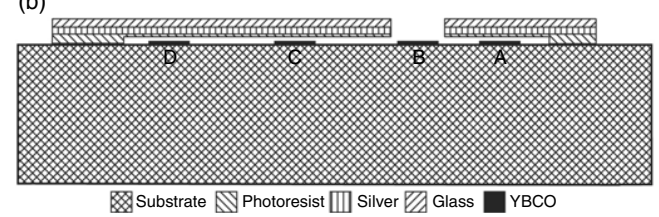

Figure 1. (a) Top and (b) side view of the test devices. The illuminated device and the neighbouring devices are shown together with the shadow mask.

Radiation blocking was achieved in a flip-chip configuration. The reflecting mask was made of a $250 \mathrm{~nm}$ thick sputtered silver layer on $0.1 \mathrm{~mm}$ glass, so that the IR transmittance was decreased by $99 \%$. Then, using the standard lithography process, a $25 \mu \mathrm{m}$ wide groove was opened in the reflecting layer. A $1.4 \mu \mathrm{m}$ thick photoresist layer was spun, and a larger window was opened so that the mask was free standing on top of the devices, eliminating any parasitic thermal or electrical contacts that could affect the measurements. Finally, the groove was aligned and fixed on top of the source bolometer as shown in figures 1(a) and (b). The contact paths and pads were coated with a sputtered gold layer so that the YBCO contact paths with nonzero resistance at the operating temperatures were shorted, assuring that the generated response was only due to the bridges. The effective lengths of the bridges facing the direct thermal coupling were $0.5 \mathrm{~mm}$, so that the lateral thermal conductance dominated over the longitudinal thermal conductance of the devices. The responses of the samples were measured using a DC bias current, $I_{\text {bias }}$, in the 4-probe configuration using an automated low noise characterization setup. The temperature of the substrate was controlled with a maximum $20 \mathrm{mK}$ deviation from the target temperature using a liquid nitrogen dewar (Janis VPF-475) and a software PID controller. The phase and magnitude of the optical response of the devices were measured with an SR 850 DSP lock-in amplifier, the input of which was amplified with an ultra-low noise preamplifier (Stanford SR 570). As a radiation source, an electrically modulated, fibre coupled IR laser diode with a wavelength of $850 \mathrm{~nm}$, and $12 \mathrm{~mW}$ power was used [7]. The system is capable of measuring all four devices in one cooling cycle without altering the electrical or thermal contacts, or the optical setup. In all of the measurements, the magnitude of the response was at least one order of magnitude greater than the system noise.

The responses of the devices were measured versus radiation modulation frequency in the range of $1 \mathrm{~Hz}-100 \mathrm{kHz}$,

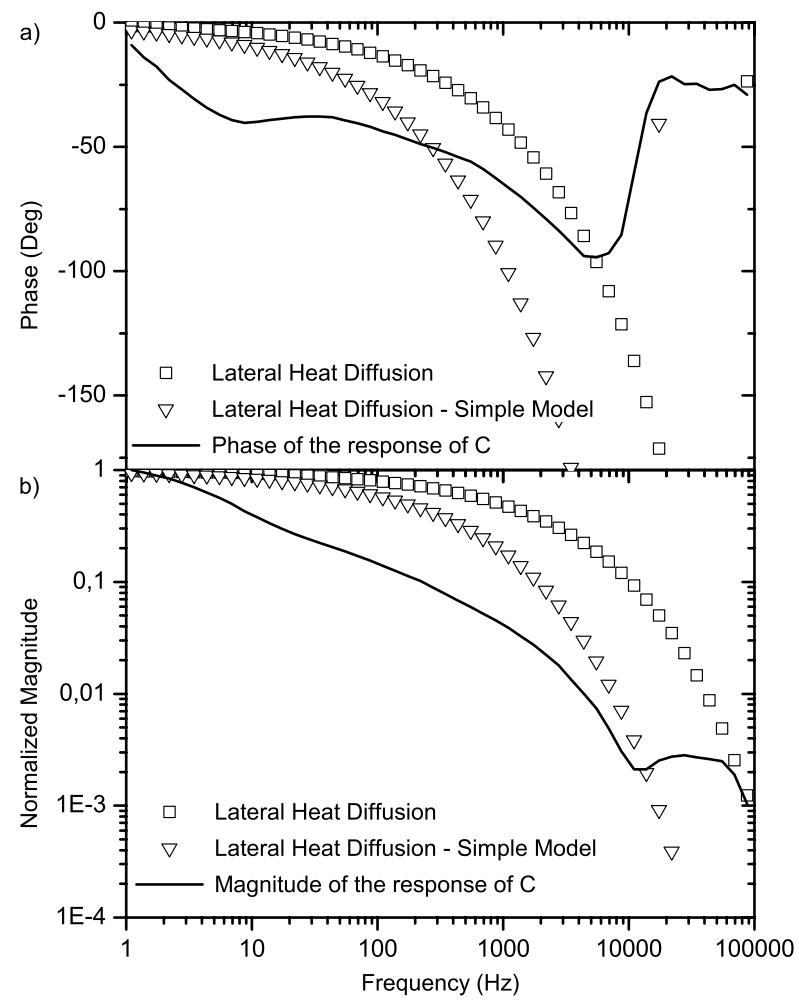

Figure 2. Measurement results of the response of device $\mathrm{C}(-)$ and thermal modelling results for the lateral heat diffusion equation (ם). $\nabla$ shows results of the simple model from [6].

limited by the lock-in amplifier. During the measurements, the temperature was fixed at three different values. First, the temperature was fixed at the middle of the superconductivity transition where the highest response magnitude was obtained $\left(T_{\mathrm{c}-\text { mid }}\right)$, then it was fixed above and below the $T_{\mathrm{c}-\text { mid }}$ to get a response magnitude approximately $10 \%$ of the maximum. These temperature values were defined as $T_{\mathrm{c}-\text { onset }}$ and $T_{\mathrm{c}-\text { zero }}$ respectively. This set of measurements was repeated for bolometers made on different substrate materials $\left(\mathrm{LaAlO}_{3}\right.$ and $\mathrm{SrTiO}_{3}$ ), for films of different thicknesses (200 and $400 \mathrm{~nm}$ ).

The measured responses of the sense devices versus the radiation modulation frequency can be investigated in two main parts: crosstalk based response and leaking laser based response. The former part is generated due to the crosstalk between the source and sense devices, and the latter part is the response generated by the leaking laser beam directly due to the imperfect blocking of the radiation by the reflecting shadow mask. For example, the response of device $\mathrm{C}$ in figures 2 and 3 is due to the crosstalk up to about $4 \mathrm{kHz}$, and is mainly due to the direct absorption of the leaking laser beam after about $10 \mathrm{kHz}$. As observed in figure 3, the phase and magnitude behaviour of the response of device $\mathrm{C}$ are the same as the source device $\mathrm{B}$ for $f \geqslant 10 \mathrm{kHz}$. For device $\mathrm{C}$, which is separated from device B by $50 \mu \mathrm{m}$, the crosstalk-free modulation frequency is around $10 \mathrm{kHz}$. Above this frequency, the coupling is expected to become negligible and the unblocked input laser radiation starts to dominate. As observed in figure 3(b), the magnitude of the response of device $\mathrm{C}$ above crosstalk-free modulation frequencies is approximately two orders smaller than that in device B, which 


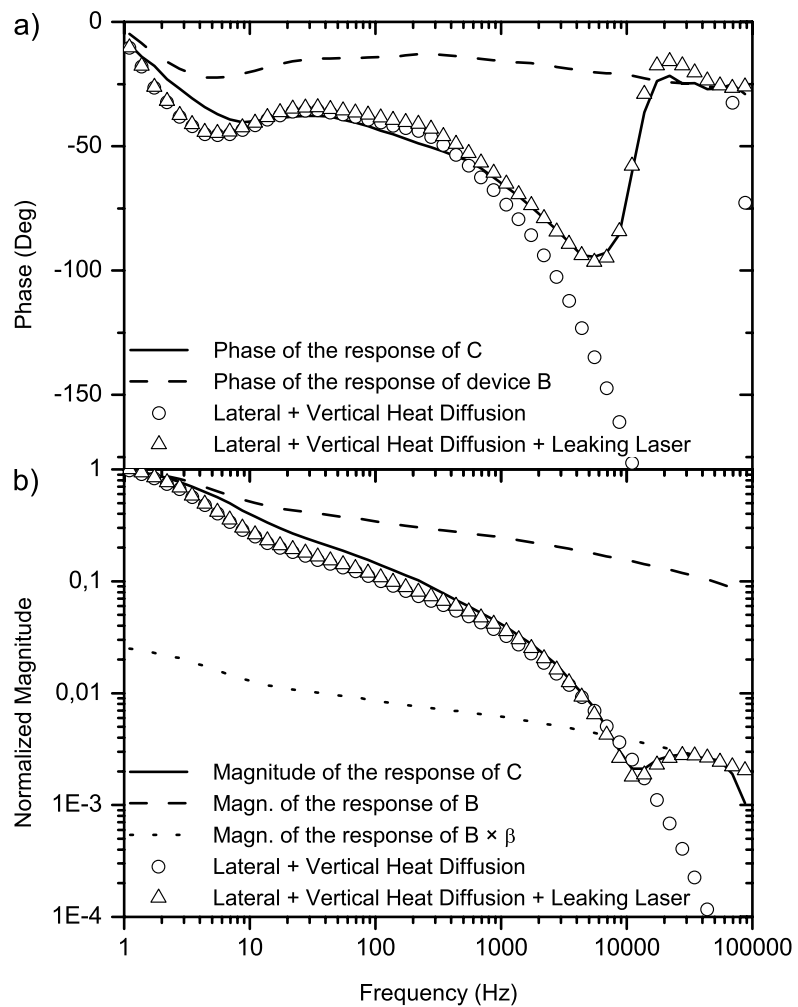

Figure 3. Measurement results of the response of device B (- - - ) and $\mathrm{C}(-)$ and analytical model without $(\mathrm{O})$ and with $(\triangle)$ leaking laser effect. $(\cdots \cdots)$ shows the magnitude of device B multiplied by the transparency $(\beta)$ of the mask.

shows that the radiation blocking of the shadow mask is more than $99 \%$.

\section{Analytical modelling of the crosstalk}

In previously reported studies $[6,8]$, we calculated the lateral thermal diffusivity $\left(D_{\mathrm{L}}\right)$ of the $\mathrm{SrTiO}_{3}$ and $\mathrm{LaAlO}_{3}$ substrate materials by only taking into account the fundamental thermal diffusion equation. That approach is valid for quick design purposes, such as deciding on the device dimensions and the operating modulation frequency. In this section, we present a more robust model that can also be used to explain the observed response behaviours of the characterized devices. The model has three main parts: (i) effect of the lateral heat diffusion, (ii) effect of the vertical heat diffusion, and (iii) effect of the leaking input laser through the mask. Basically, the previously reported lateral thermal diffusion parameter takes into account all these three parts up to the frequency ranges that the crosstalk is not negligible. In this paper, we do not use the previously defined lateral thermal diffusivity, but use the bulk thermal diffusivity of the substrate material. For comparison purposes, the results of the simple model with lateral diffusivity values is also given in section 3.1 .

\subsection{Heat diffusion on the surface of the substrate}

Assuming only lateral heat propagation in the substrate, the spatial variation of the response at distance $x$ away from a single pixel bolometer has been formulated as [11, 12]

$$
\frac{T(x, f)}{T_{0}}=\underbrace{\exp \left[-\sqrt{\frac{\pi f}{D}} x\right]}_{\text {magnitude }} \exp [\underbrace{\left.-\sqrt{\frac{\pi f}{D}} x j\right]}_{\text {phase }}
$$

where $D$ is the thermal diffusivity of the substrate material, $f$ is the modulation frequency, $x$ is the distance from the bolometer, and $T / T_{o}$ is the spatial and frequency dependent variation of the temperature in the substrate. The points represented with ( $\square$ ) in figure 2 show the results of (1). It is seen that the lateral heat propagation equation is not adequate for explaining the experimental data shown with straight lines in figure 2. However, as in [6], for simple design purposes, a new term called lateral thermal diffusivity $\left(D_{\mathrm{L}}\right)$ was introduced and used in (1). The result of this approach is shown with $(\nabla)$ in figure 2. As shown, it gives a better approximation than the use of bulk diffusivity of the substrate in the lateral heat diffusivity approximation.

\subsection{Heat diffusion in the bulk}

In previously reported single pixel models [3], we showed that for a $1 \mathrm{~mm}$ thick $\mathrm{SrTiO}_{3}$ substrate, the substrate-cold head Kapitza boundary resistance is effective up to a modulation frequency of $4 \mathrm{~Hz}$. In figure 3, it is shown that in this array configuration there is also a knee frequency around $4 \mathrm{~Hz}$ in the crosstalk based response, which is due to the effect of the Kapitza resistance. Thus we cannot neglect the effects of the vertical propagation and the interfaces. However, (1) assumes that heat only propagates in the surface of the substrate material. Hence, another term has to be added to (1) to be able to take the thermal parameters caused by the bulk and the interfaces into account as follows:

$$
r_{\mathrm{v}-\mathrm{C}}(f)=\underbrace{\exp \left[-(1+j) \sqrt{\frac{\pi f}{D}} x\right]}_{\text {lateral heat diffusion term }} \times \underbrace{\left(r_{\mathrm{v}-\mathrm{B}}(f)\right)^{\alpha}}_{\begin{array}{c}
\text { vertical heat } \\
\text { diffusion term }
\end{array}}
$$

where $r_{\mathrm{v}-\mathrm{C}}(f)$ is the measured crosstalk based response of the sense pixel $\mathrm{C}, r_{\mathrm{v}-\mathrm{B}}(f)$ is the experimental data of the device $\mathrm{B}$ and $\alpha$ is the term that accounts for the amount of crosstalk delay caused by the substrate and the interfaces. Basically, as crosstalk lag decreases, i.e. stronger crosstalk is observed, the exponential $\alpha$ decreases. This is an expected result because as the crosstalk between the devices increases, the lateral heat diffusion becomes more dominant, and the contribution of the deeper bulk and interfaces decreases. The result of (2) is shown with $O$ in figure 3.

For a rough estimation of the $\alpha$ parameter, a relation between the lateral thermal diffusivity is found based on the $D_{\mathrm{L}}$ values given in [6] and [8] and the $\alpha$ values used in (2). As given in table $1, \alpha \times D_{\mathrm{L}}$ is equal to 0.05 for $\mathrm{SrTiO}_{3}$ substrate and 0.11 for $\mathrm{LaAlO}_{3}$ substrate. Thus, once the lateral thermal diffusivity value of a device is known for a specific substrate, the $\alpha$ parameter can be estimated for the array. In section 4 , the use of the parameter $\alpha$ is shown with the application to devices with various physical parameters. 
Analytical modelling of the thermal crosstalk in ETB arrays

Table 1. The parameters used in the application of the thermal model to the test devices.

\begin{tabular}{rlllllllll}
\hline No & Substrate & $\begin{array}{l}D^{\mathrm{a}} \\
\left(\mathrm{cm}^{2} \mathrm{~s}^{-1}\right)\end{array}$ & $\begin{array}{l}D_{\mathrm{L}}^{\mathrm{b}} \\
\left(\mathrm{cm}^{2} \mathrm{~s}^{-1}\right)\end{array}$ & $\begin{array}{l}T \\
(\mathrm{~K})\end{array}$ & $\begin{array}{l}t^{\mathrm{c}} \\
(\mathrm{nm})\end{array}$ & \multicolumn{1}{c}{$\begin{array}{l}x^{\mathrm{c}} \\
(\mu \mathrm{m})\end{array}$} & $\beta^{\mathrm{c}}$ & $\alpha$ & $D_{\mathrm{L}} \times \alpha$ \\
\hline 1 & $\mathrm{SrTiO}_{3}$ & 0.12 & 0.028 & $T_{\mathrm{c}}$ & 200 & 30 & 0.010 & 1.9 & 0.053 \\
2 & $\mathrm{SrTiO}_{3}$ & 0.12 & 0.027 & $T_{\mathrm{c}}$ & 200 & 50 & 0.010 & 1.9 & 0.051 \\
3 & $\mathrm{SrTiO}_{3}$ & 0.12 & 0.030 & $T_{\mathrm{c}-\text { zero }}$ & 200 & 150 & 0.010 & 1.75 & 0.052 \\
4 & $\mathrm{SrTiO}_{3}$ & 0.12 & 0.026 & $T_{\mathrm{c}}$ & 200 & 150 & 0.010 & 1.9 & 0.049 \\
5 & $\mathrm{SrTiO}_{3}$ & 0.12 & 0.022 & $T_{\mathrm{c}-\text { onset }}$ & 200 & 150 & 0.022 & 2.1 & 0.046 \\
6 & $\mathrm{SrTiO}_{3}$ & 0.12 & 0.025 & $T_{\mathrm{c}}$ & 400 & 30 & 0.009 & 2.2 & 0.055 \\
7 & $\mathrm{SrTiO}_{3}$ & 0.12 & 0.026 & $T_{\mathrm{c}}$ & 400 & 50 & 0.008 & 2.2 & 0.057 \\
8 & $\mathrm{SrTiO}_{3}$ & 0.12 & 0.024 & $T_{\mathrm{c}}$ & 400 & 150 & 0.009 & 2.2 & 0.052 \\
9 & $\mathrm{LaAlO}_{3}$ & 0.32 & 0.086 & $T_{\mathrm{c}-\text { zero }}$ & 200 & 50 & 0.015 & 1.3 & 0.11 \\
10 & $\mathrm{LaAlO}_{3}$ & 0.32 & 0.078 & $T_{\mathrm{c}}$ & 200 & 50 & 0.009 & 1.5 & 0.11 \\
11 & $\mathrm{LaAlO}_{3}$ & 0.32 & - & $T_{\mathrm{c}-\text { onset }}$ & 200 & 50 & 0.016 & 1.6 & - \\
\hline
\end{tabular}

${ }^{\mathrm{a}} \mathrm{SrTiO}_{3}$ : from [9], $\mathrm{LaAlO}_{3}: 0.55$ from [10], 0.28 from [3].

b $\mathrm{SrTiO}_{3}$ : from [6], $\mathrm{LaAlO}_{3}$ : from [8].

${ }^{\mathrm{c}} t$ : film thickness, $x$ : distance from the source device, $\mathrm{B}, \beta$ : transmittance of the mask.

\subsection{Leaking input laser effect}

As seen in figure 3, the phase and magnitude of the crosstalk response that are shown with $\bigcirc$ continuously decrease. However, after some frequency, the phase of the experimental data shown with the straight line recovers and it converges to that of device $\mathrm{B}$ and likewise, the magnitude of device $\mathrm{C}$ recovers to the magnitude of $\mathrm{B}$ scaled by the transparency of the reflecting mask. Thus, after some frequency, the response caused by the input laser becomes dominant. The input laser leaks to device $\mathrm{C}$ through the reflecting mask. If we add this leaking term to the crosstalk response, we get the following relation whose plot is shown with $\triangle$ in figure 3:

$$
\begin{aligned}
& r_{\mathrm{v}-\mathrm{C}}(f)=\underbrace{\exp \left[-(1+j) \sqrt{\frac{\pi f}{D}} x\right]}_{\text {lateral heat diffusion term }} \times \underbrace{\left(r_{\mathrm{v}-\mathrm{B}}(f)\right)^{\alpha}}_{\begin{array}{c}
\text { vertical heat } \\
\text { diffusion term }
\end{array}} \\
& +\underbrace{r_{\mathrm{v}-\mathrm{B}}(f) \times \beta}_{\text {leaking laser term }} .
\end{aligned}
$$

In our experiments, the transparency of the reflecting mask was of the order of $1 \%$, as shown in table 1 . Thus at low frequencies, where the crosstalk is dominant, the effect of the leaking laser term is negligible.

\section{Application of the model to the test devices}

In the previous section, we chose an arbitrary sample's data and demonstrated the derivation and physical basis of the model. We have already reported the qualitative analysis of the effects of the physical parameters of the devices on the thermal crosstalk between the devices of an array [6,13], and hence we will not get into the details here. In this section, we present the results of applying the model to devices of various physical parameters and verify its validity and test its range of applicability. We have applied the model to the 11 devices listed in table 1 and obtained a fairly good fit with the experimental results.

\subsection{Device separation}

The distance between the source and sense pixels is one of the main parameters that affect the crosstalk between the devices.

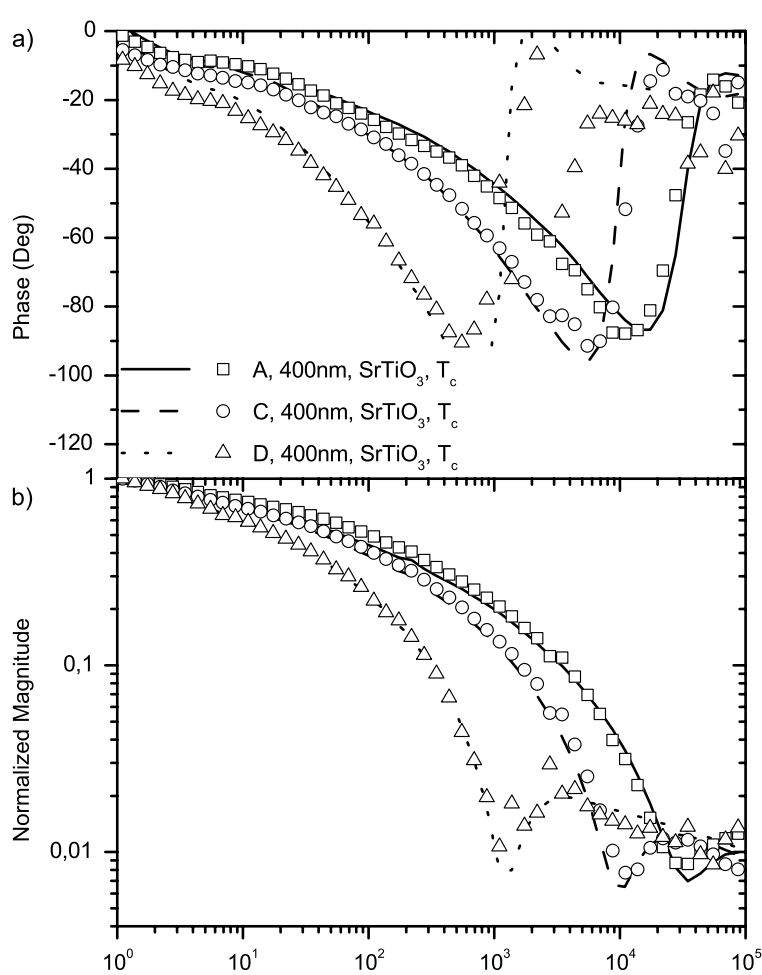

Figure 4. Verification of the model with sense devices at different distances from the source device (table 1 rows $6,7,8$ ). The scatter plots show the experimental data, the line plots show the results of the analytical model.

As the distance between the devices starts to be comparable to the thermal diffusion length at a specific frequency, the response curves of bolometers A, C, and D start to diverge from each other as shown in 4. Eventually, after the modulation frequency becomes high enough to cease the coupling, the devices again converge to the response of the input device, B, due to the leaking laser beam as discussed earlier.

In figure 4 we see the effect of the separation, $x$, between the devices on the crosstalk. When we apply the model to devices 5,6 , and 7 on the same substrate with different $x$ values, we see that the model fits well by only varying $x$ in (3), while other parameters are constant as listed in table 1 . 


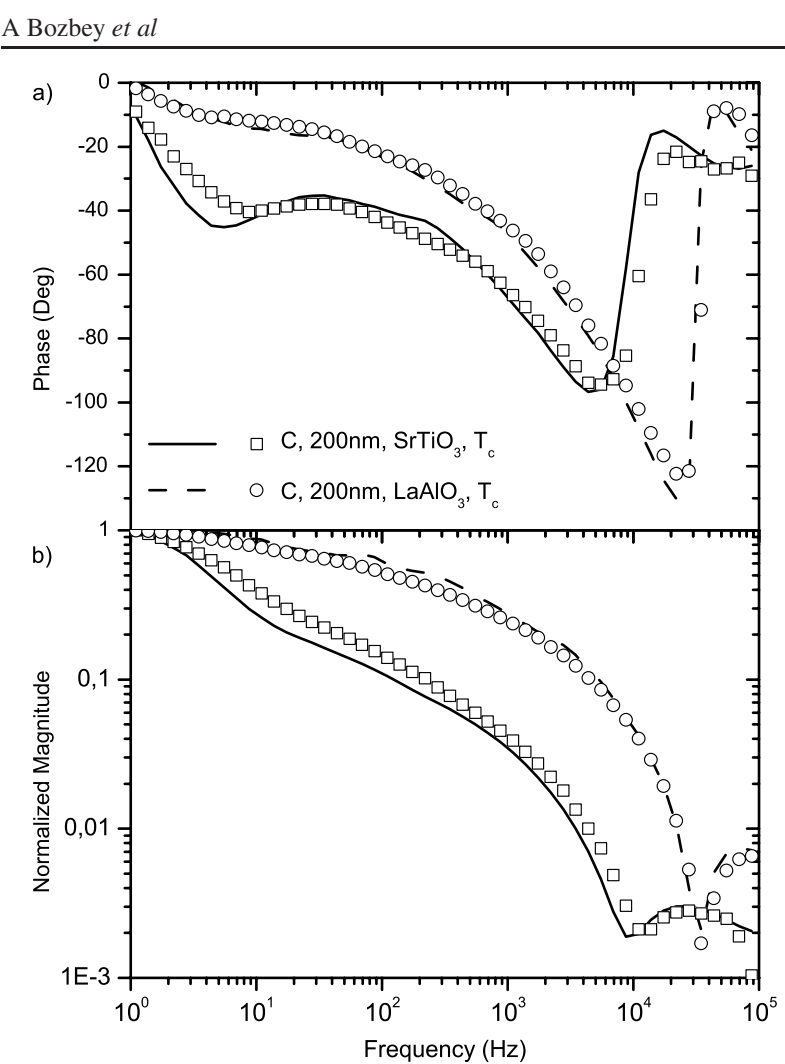

Figure 5. Verification of the model with devices made on different substrate materials (table 1 rows 2,10 ). The scatter plots show the experimental data, the line plots show the results of the analytical model.

\subsection{Substrate material}

The thermal diffusivity of the substrate material has a major effect on the crosstalk response of the bolometers. As demonstrated in figure 5, it is shown that the model is able to explain the crosstalk response for the two different types of substrate. As listed in the table 1, the only different physical parameter between devices 2 and 10 is the diffusivity values of the substrate materials. Since the substrate has changed, the $\alpha$ parameter in (3) should be changed. $\mathrm{LaAlO}_{3}$ has a lower $\alpha$ value than that of $\mathrm{SrTiO}_{3}$ since the diffusivity of $\mathrm{LaAlO}_{3}$ is higher leading to higher crosstalk as observed, and the thermal parameters in the vertical direction, such as the interfaces, have less affect on the response. In addition, the absorbtivities of the $\mathrm{LaAlO}_{3}$ and $\mathrm{SrTiO}_{3}$ substrates at $850 \mathrm{~nm}$ wavelength are $23 \%$ and $13 \%$ respectively [14]. Thus, the ratio of the absorbed radiation by the YBCO film and the substrate material is higher in $\mathrm{SrTiO}_{3}$, which affects the amount of crosstalk between the devices.

\subsection{Film thickness}

In figure 6 , it is shown that the model also fits very well to the crosstalk between the devices made of thicker films. Devices 2 and 7 are used to test the model. In this fitting, the only parameter that has changed is the $\alpha$ parameter. As shown in table 1 , the thick film based device (device 7) has a relatively greater $\alpha$ value. We associate this with the ratio of the absorbed radiation by the film and that of the substrate material. As the

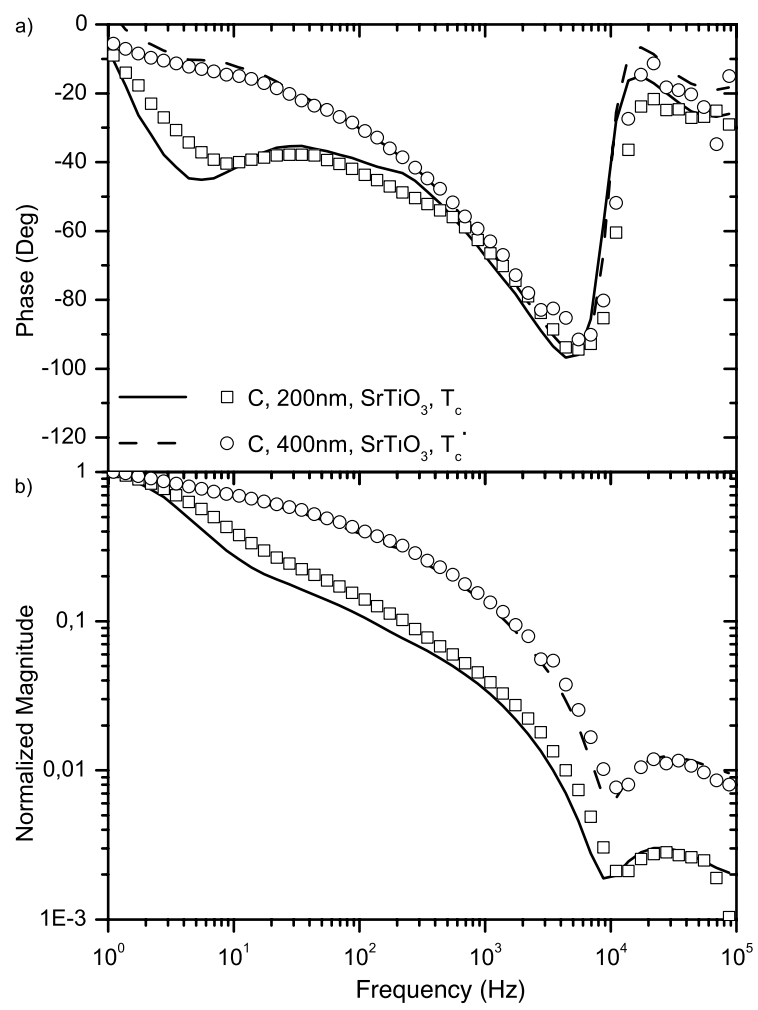

Figure 6. Verification of the model with devices made of different film thicknesses (table 1 rows 2, 7). The scatter plots show the experimental data, the line plots show the results of the analytical model.

film thickness is increased the amount of radiation absorbed by the film increases. Thus, the ratio of the absorbed radiation by the YBCO film and the substrate material is higher in the thicker films. A similar variation in the $\alpha$ parameter is observed for the substrate material effect.

\subsection{Bias temperature}

We have already reported the qualitative analysis of the temperature dependence of the thermal crosstalk between the source and sense pixels elsewhere [6]. There, we showed that although the source pixels responses do not have a strong temperature dependence, the sense pixels' responses had a strong dependence on the temperature. Thus, the temperature dependent responses of devices $\mathrm{A}, \mathrm{C}$, and $\mathrm{D}$ are mainly associated with the superconductivity transition dependent crosstalk between the devices.

In figure 7 , it is shown that the model is able to fit the response of the devices at different temperatures. Table 1 shows the parameters of the devices and the parameters used in the model. We have measured the devices made on both $\mathrm{LaAlO}_{3}(9,10,11)$ and $\mathrm{SrTiO}_{3}(3,4,5)$ and obtained similar response behaviours. Figure 7 shows the experimental results and fitting curves of devices 9, 10, and 11 . The temperature dependence of the crosstalk has been investigated in detail elsewhere [6]. There, we concluded that as the bias temperature is decreased, the crosstalk between the devices increases. Based on the observations in section 4.2, as the 


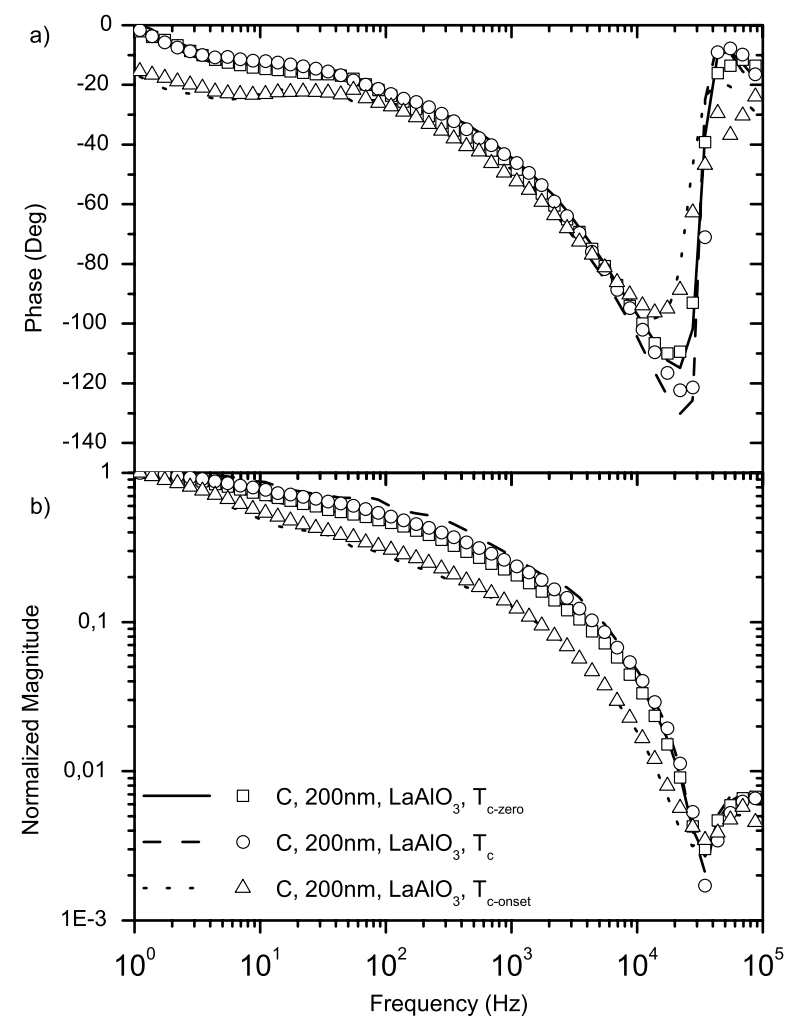

Figure 7. Verification of the model with devices under different bias temperatures (table 1 rows $9,10,11$ ). The scatter plots show the experimental data, the line plots show the results of the analytical model.

crosstalk is increased the $\alpha$ parameter decreases, and in table 1 we see that this result is valid in the temperature effect as well.

\section{Conclusion}

An analytical model has been proposed and verified to explain the dependence of the crosstalk on the physical parameters of the devices, bias temperature, and the modulation frequency. We have shown that if the modulation frequency is high enough, in addition to the crosstalk based response, the leaking laser term, due to imperfect blocking of the mask, should be taken into account.

The model is suitable for detailed design and implementations of edge-transition bolometer arrays. It requires experimental data from a single pixel device to be able to extend the lateral heat diffusion equation to a 3D model. At first sight, the requirement of single pixel response data may seem like a weak point of the model, but obtaining the response data for a single pixel device is trivial, and it is not necessary to obtain the data for each and every device. For different substrate materials and film thicknesses the data can be tabulated. In addition, to make the model free of experimental single pixel device data, previously reported single pixel bolometer models can be used. We think that this only complicates the model without gaining much in terms of practical use.

Apart from the conventional device parameters, such as the device dimensions, thermal capacitance or conductances, the model introduces a new parameter, $\alpha$. As shown above, $\alpha$ is a measurable parameter, it is a measure of the amount of crosstalk and has a systematic dependence on the other device parameters. We observed that as the crosstalk is increased the $\alpha$ parameter decreases, which is verified by the substrate effect and temperature effect. As the ratio of the absorption of the film to substrate increases the $\alpha$ parameter decreases, which is verified by the substrate effect and film thickness effect. As shown in the previous sections, $\alpha \times D_{\mathrm{L}}$ has a constant value for a specific substrate, and this is in agreement with the two observations above.

\section{References}

[1] Adam A, Gauge A, Ulysse C, Kreisler A and Boulanger C 2003 Three-temperature model for hot electron superconducting bolometers based on high- $T_{c}$ superconductor for terahertz applications IEEE Trans. Appl. Supercond. 13 (12)

[2] Klokov A Y, Galkina T I and Plotnikov A F 1998 Response of a $\mathrm{YBaCuO} / \mathrm{MgO}$ bolometer structure at high levels of laser excitation. nonlinear model and experiment Phys. Solid State 40 (2)

[3] Fardmanesh M 2001 Analytic thermal modelling for DC-to-midrange modulation frequency responses of thin-film high- $T_{c}$ superconductive edge-transtition bolometers Appl. Opt. 40 1080-8

[4] Gaugue A, Teste P, Delerue J, Gensbittel A, Luca A D, Kreisler A, Voisin F, Klisnick G and Redon M 2001 $\mathrm{YBaCuO}$ mid-infrared bolometers: substrate influence on inter-pixel crosstalk IEEE Trans. Appl. Supercond. 11 766-9

[5] Delerue J, Gaugue A, Teste P, Caristan E, Klisnick G, Redon M and Kreisler A 2003 YBCO mid-infrared bolometer arrays IEEE Trans. Appl. Supercond. 13 176-9

[6] Bozbey A, Fardmanesh M, Schubert J and Banzet M 2006 Superconductivity transition dependence of the thermal crosstalk in YBCO edge transition bolometer arrays IEEE Trans. Appl. Supercond. 16 (1)

[7] Bozbey A, Fardmanesh M, Askerzade I, Banzet M and Schubert J 2003 Effects of the superconductivity transition on the response of YBCO edge transition bolometers Supercond. Sci. Technol. 16 1554-8

[8] Bozbey A, Fardmanesh M, Schubert J and Banzet M 2005 YBCO kenar gecis bolometre dizilerinde termal baglasimin speriletkenlik gecisi ve aygit parametrelerine bagimliligi. (Bolu, Turkey): III. Ulusal Yuksek Sicaklik Superiletkenler Seтроzуити

[9] Oppenheim U P, Katz M, Koren G, Polturak E and Fishman M R 1991 High temperature superconducting bolometer Physica C 178 26-8

[10] Nahum S V M and Richards P L 1991 Thermal boundary resistance of $\mathrm{YBa}_{2} \mathrm{Cu}_{3} \mathrm{O}_{7-\delta}$ films Appl. Phys. Lett. $592034-6$

[11] Dwir B and Pavuna D 1992 A sensitive $\mathrm{YBaCuO}$ thin film bolometer with ultrawide wavelength response J. Appl. Phys. 72 3855-61

[12] Hu Q and Richards P L 1998 Design analysis of a high $T_{c}$ superconducting microbolometer Appl. Phys. Lett. $552444-6$

[13] Bozbey A, Fardmanesh M, Schubert J and Banzet M 2006 Substrate and pattern dependence of the thermal crosstalk in YBCO edge transition bolometer arrays IEEE Trans. Appl. Supercond. accepted

[14] Bozbey A 2003 YBCO edge transition bolometers: effect of superconductivity transition on the phase and magnitude of the response Master's Thesis Bilkent University, Dept of EE 